\title{
LHC OPTICS AND ELASTIC SCATTERING MEASURED BY THE TOTEM EXPERIMENT
}

\author{
F. Nemes, for the TOTEM Collaboration ${ }^{1,2} a$ \\ ${ }^{1}$ CERN, Geneva, Switzerland \\ ${ }^{2}$ Wigner RCP, H-1121 Budapest XII, Konkoly-Thege 29-33, Hungary
}

\begin{abstract}
The TOTEM experiment at the LHC has measured proton-proton elastic scattering in dedicated runs at $\sqrt{s}=7$ and 8 TeV centre-of-mass LHC energies. The proton-proton total cross-section $\sigma_{\text {tot }}$ has been derived for both energies using a luminosity independent method. TOTEM has excluded a purely exponential differential cross-section for elastic proton-proton scattering with significance greater than $7 \sigma$ in the $|t|$ range from 0.027 to $0.2 \mathrm{GeV}^{2}$ at $\sqrt{s}=8 \mathrm{TeV}$.
\end{abstract}

\section{Introduction}

The TOTEM (TOTal cross section, Elastic scattering and diffraction dissociation Measurement at the LHC) experiment has been designed to measure the total proton-proton (pp) cross-section, elastic scattering and diffractive processes at the LHC [1], see Fig. 1.

The experimental apparatus of TOTEM is composed of three subdetectors: the Roman Pots (RP), the T1 and T2 inelastic forward telescopes. The detectors are placed symmetrically on both sides of the Interaction Point 5 (IP5), which is shared with the CMS experiment.

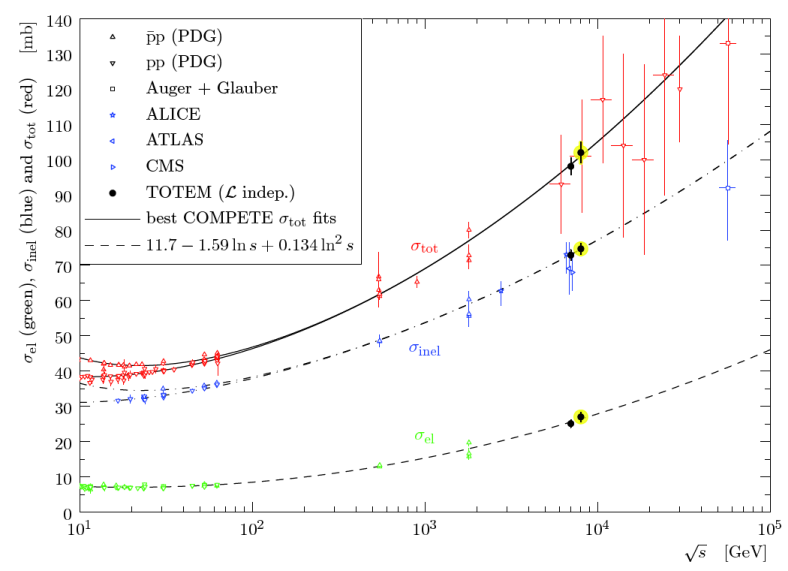

Figure 1: A compilation of total, inelastic and elastic pp cross-section measurements. The black points indicate the TOTEM measurements at $\sqrt{s}=7$ and $8 \mathrm{TeV}$ using the luminosity independent method 2,7$]$.

\footnotetext{
${ }^{a}$ E-mail: fnemes@cern.ch
} 
The RPs are moveable beam-pipe insertions, hosting edgeless silicon detectors to detect leading protons scattered at very small angles. In order to maximize the acceptance of the experiment for elastically scattered protons, the RPs are able to approach the beam center to a transverse distance as small as $1 \mathrm{~mm}$. The alignment of the RPs is optimized by reconstructing common tracks going through the overlap between the vertical and horizontal RPs 1, 8 .

Before the LHC long shutdown one (LS1) the RPs, used for measurements, were located at distances of 215-220 m from IP5 1]. The actual layout, i.e., after the LHC LS1, is different in RP location and quantity. The RP stations previously installed at $\pm 147 \mathrm{~m}$, from IP5, have been relocated to $\pm 210 \mathrm{~m}$. Moreover, two newly designed horizontal RPs have been installed between the two units of the $\pm 220 \mathrm{~m}$ station 9,10$]$.

\section{Elastic scattering and total cross-section $\sigma_{\text {tot }}$ measurements}

For each tagged elastic event the four-momentum transfer squared $t$ is reconstructed using the LHC optical functions, characterized with the so-called betatron amplitude at IP5 $\beta^{*}[1]$. The TOTEM experiment developed a novel experimental method to estimate the optical functions at the RP locations, using the measured elastically scattered protons $11-14$, see Fig. 2 .
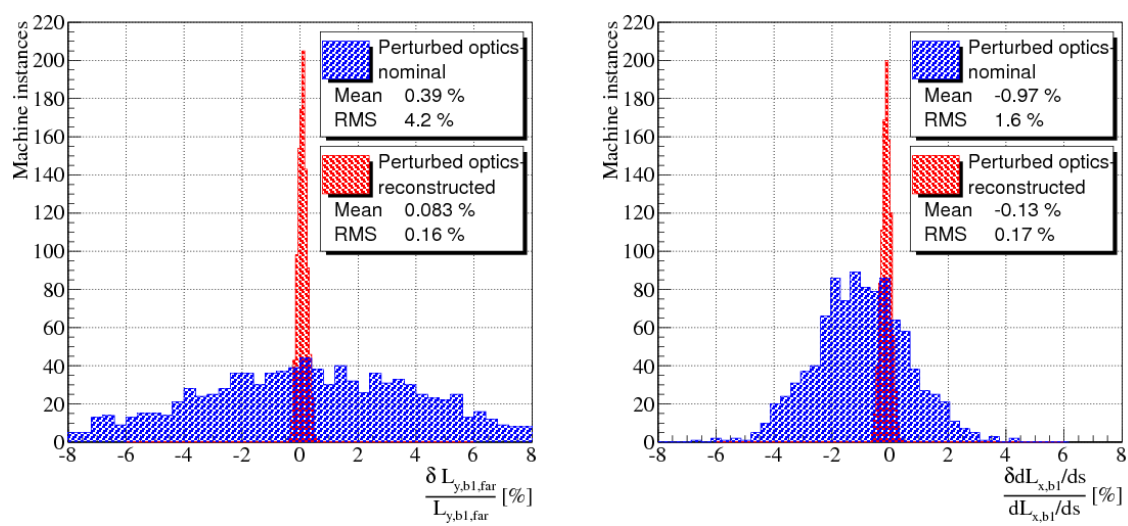

Figure 2: The MC error distribution of $\beta^{*}=3.5 \mathrm{~m}$ optical functions $L_{y}$ and $\mathrm{d} L_{x} / \mathrm{d} s$ for Beam 1 at $\sqrt{s}=7 \mathrm{TeV}$, before and after optics estimation.

The total inelastic rate $N_{\text {inel }}$, measured by the T1 and T2 telescopes, and the total nuclear elastic rate $N_{\text {el }}$ with its extrapolation to zero four-momentum transfer squared $t=0$ are combined with the optical theorem to obtain the 
total cross-section in a luminosity, $\mathcal{L}$, independent way

$$
\sigma_{\text {tot }}=\left.\frac{16 \pi}{1+\rho^{2}} \cdot \frac{\mathrm{d} N_{\mathrm{el}}}{\mathrm{d} t}\right|_{t=0} \cdot\left(N_{\mathrm{el}}+N_{\text {inel }}\right)^{-1} .
$$

The measured ratio of the elastic and inelastic rates $N_{\mathrm{el}} / N_{\text {inel }}$ allows for the determination of the elastic and inelastic cross-sections as well [1, 14].

The TOTEM experiment determined the total pp cross-section at $\sqrt{s}=$ $7 \mathrm{TeV}$ using the luminosity independent method [2], which was shown to be consistent with the total cross-sections measured in independent ways, see Table 1 The elastic and inelastic cross-sections were found to be $\sigma_{\mathrm{el}}=25.1 \pm 1.1 \mathrm{mb}$ and $\sigma_{\text {inel }}=72.9 \pm 1.5 \mathrm{mb}$.

The measurement was repeated at $\sqrt{s}=8 \mathrm{TeV}$, yielding to $\sigma_{\text {tot }}=101.7 \pm$ $2.9 \mathrm{mb}, \sigma_{\mathrm{el}}=27.1 \pm 1.4 \mathrm{mb}$ and $\sigma_{\text {inel }}=74.7 \pm 1.7 \mathrm{mb} 3$. A compilation of the results is shown in Fig. 1, which also demonstrates that the observed cross-sections are in agreement with low-energy data and cosmic ray results as well 4 [7].

\begin{tabular}{|c|c|c|c|c|}
\hline Method & $\mathcal{L}$ independent 2 & $\underline{12}$ & $\underline{13}$ & \\
\hline$\sigma_{\text {tot }}[\mathrm{mb}]$ & $98.0 \pm 2.5$ & $98.3 \pm 2.8$ & $98.6 \pm 2.2$ & $99.1 \pm 4.3$ \\
\hline
\end{tabular}

Table 1: The total cross-section $\sigma_{\text {tot }}$ results measured by the TOTEM experiment at $\sqrt{s}=$ $7 \mathrm{TeV}$ with four different methods.

Thanks to a very-high statistics $\beta^{*}=90 \mathrm{~m}$ data set at $\sqrt{s}=8 \mathrm{TeV}$ energy, the TOTEM experiment has excluded a purely exponential elastic pp differential cross-section [15]. The exclusion's significance is greater than $7 \sigma$ in the $|t|$ range from 0.027 to $0.2 \mathrm{GeV}^{2}$, see Fig. 3. Using refined parametrizations for the extrapolation to the optical point, $t=0$, yields total cross-section values $\sigma_{\text {tot }}=101.5 \pm 2.1 \mathrm{mb}$ and $\sigma_{\text {tot }}=101.9 \pm 2.1 \mathrm{mb}$, compatible with the previous TOTEM measurement. The TOTEM experiment performed its first measurement of elastic scattering in the Coulomb-nuclear interference (CNI) region 16 . The data have been collected at $\sqrt{s}=8 \mathrm{TeV}$ with a special beam optics of $\beta^{*}=1000 \mathrm{~m}$. The $\rho$ parameter was for the first time at LHC extracted via the Coulomb-nuclear interference, and was found to be $\rho=0.12 \pm 0.03$.

\section{Conclusions}

The TOTEM experiment has measured elastic pp scattering at $\sqrt{s}=7$ and $8 \mathrm{TeV}$. The total, elastic and inelastic cross-sections have been derived for both energies using a luminosity independent method. TOTEM has excluded a purely exponential differential cross-section for elastic pp scattering with significance greater than $7 \sigma$ in the $|t|$ range from 0.027 to $0.2 \mathrm{GeV}^{2}$ at $\sqrt{s}=$ $8 \mathrm{TeV}$. Using $\beta^{*}=1000 \mathrm{~m}$ optics at $\sqrt{s}=8 \mathrm{TeV}$ energy the $\rho$ parameter was for the first time at LHC extracted via the Coulomb-nuclear interference. 


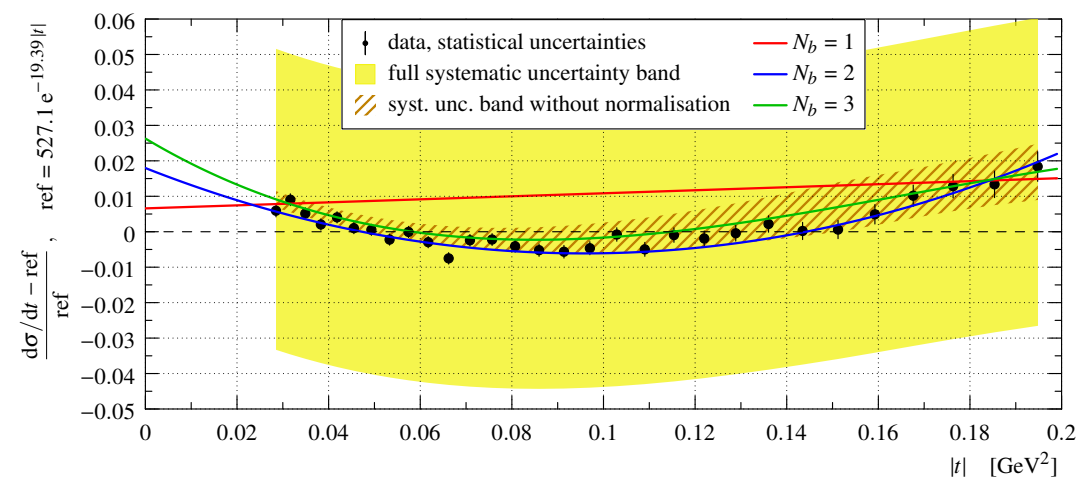

Figure 3: Differential cross-section measured at $\sqrt{s}=8 \mathrm{TeV}$ LHC energy plotted as relative difference from a reference exponential. The black dots represent data points with statistical uncertainty bars 15 .

\section{Acknowledgement}

This work was supported by the institutions involved in the TOTEM collaboration and partially also by NSF (US), the Magnus Ehrnrooth Foundation (Finland), the Waldemar von Frenckell Foundation (Finland), the Academy of Finland, the Finnish Academy of Science and Letters (The Vilho, Yrjo and Kalle Vaisa la Fund) as well as by the Hungarian OTKA grant NK 101438.

[1] G. Anelli et al. [TOTEM Coll.], JINST 3 (2008) S08007.

[2] G. Antchev et al. [TOTEM Coll.], Europhys. Lett. 101, 21004 (2013).

[3] G. Antchev et al. [TOTEM Coll.], Phys. Rev. Lett. 111 (2013) 1, 012001.

[4] B. Abelev et al. [ALICE Coll.], Eur. Phys. J. C 73 (2013) 6, 2456

[5] S. Chatrchyan et al. [CMS Coll.], Phys. Lett. B 722 (2013) 5

[6] ATLAS Collaboration, Nature Commun. 2 (2011) 463.

[7] K. A. Olive et al. [PDG Coll.], Chin. Phys. C 38 (2014) 090001.

[8] G. Antchev et al. [TOTEM Coll.], Europhys. Lett. 95 (2011) 41001

[9] TOTEM Coll. [TOTEM Coll.], CERN-LHCC-2013-009, LHCC-P-007.

[10] M. G. Albrow [CMS and TOTEM Coll.], PoS DIS 2015 (2015) 064.

[11] G. Antchev et al. [TOTEM Coll.], New J. Phys. 16 (2014) 103041

[12] G. Antchev et al. [TOTEM Coll.], Europhys. Lett. 96 (2011) 21002

[13] G. Antchev et al. [TOTEM Coll.], Europhys. Lett. 101 (2013) 21002.

[14] G. Antchev et al. [TOTEM Coll.], Europhys. Lett. 101 (2013) 21003.

[15] G. Antchev et al. [TOTEM Coll.], Nucl. Phys. B 899 (2015) 527

[16] G. Antchev et al. [TOTEM Coll.], CERN-PH-EP-2015-325 